\title{
Design and Fabrication of Epicyclic Gear Box
}

\author{
S. Senthil Kumar ${ }^{1}$, J.S. Athreya ${ }^{2}$, E. Ambrish Sharma ${ }^{3}$, C. Dinesh ${ }^{4}$ \\ Department of Mechanical Engineering, RMKCET ${ }^{1,2,3,4}$
}

\begin{abstract}
Planetary Gear Trains are extensively used for the power transmission and are the most critical component. Planetary gearboxes are used frequently to match the inertias, lower the motor speed, boost the torque, and at the same time provide a sturdy mechanical interface for pulleys, cams, drums and other mechanical components. This paper presents the advantages of planetary gear systems over other, consideration to be given while determining the reduction ratios of gear box, minimum and maximum reductions per stage of planetary gear pairs. Also the case study to determine the reduction ratio of two stage gear box is discussed. The comparison of planetary gear system over helical gears systems is done on the basis of volume, weight and torque density. An epicyclic gear train consists of three gears mounted so that the centre of one gear revolves around the centre of the other. A carrier connects the centers of the two gears and rotates to carry one gear, called the planet gear, around the other, called the sun gear. The planet and sun gears mesh so that their pitch circles roll without slip. A point on the pitch circle of the planet gear traces an epicycloid curve. In this simplified case, the sun gear is fixed and the planetary gear(s) roll around the sun gear. An epicyclic gear train can be assembled so the planet gear rolls on the inside of the pitch circle of a fixed, outer gear ring, which is called an annular gear. In this case, the curve traced by a point on the pitch circle of the planet gear is a hypocycloid. The combination of epicycle gear trains with a planet gear engaging both a sun gear and an annular gear is called a planetary gear train. In this case, the annular gear is usually fixed and the sun gear is driven.
\end{abstract}

Keywords: Epicyclic Gear Box, Planetary Gear Trains, planet gear, epicycloid curve.

\section{INTRODUCTION}

Planetary gear systems normally consist of a centrally pivoted sun gear, a ring gear, and several planet gears found between the sun gear and ring gear. Now, industrial applications demands high torque in compact (a high torque/volume) and light (a high torque/weight ratio) package. In planetary gears, torque density can be increased by adding more planets through multiple gear mesh points. This means a planetary gear with say three planets can transfer three times the torque of a similar sized fixed axis — standardll spur gear system as shown in Figure 1

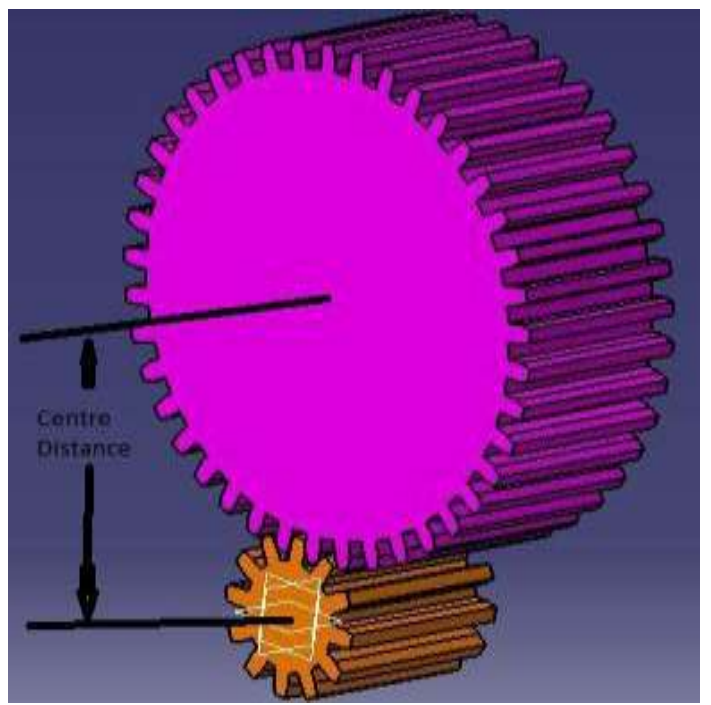

Fig 1 Meshing of two gears

The applied load to planetary gears are distributed onto multiple gear mesh points means the load is supported by $\mathrm{N}$ contacts (where $\mathrm{N}=$ number of planet gears) increasing the torsional stiffness of the gear train by factor $\mathrm{N}$. Hence it lowers the lost motion compared to similar size standard gear trains. High rotational stiffness is important for applications with positioning accuracy and repeatability requirements; especially under fluctuating loading conditions. Hence planetary gears are used for such applications in automation. Added inertia results in an additional torque/energy requirement for both acceleration and deceleration. 
The smaller gears in planetary system result in lower inertia. Compared to a same torque rating standard gearbox, it is a fair approximation to say that the planetary gearbox inertia is smaller by the square of the number of planets.

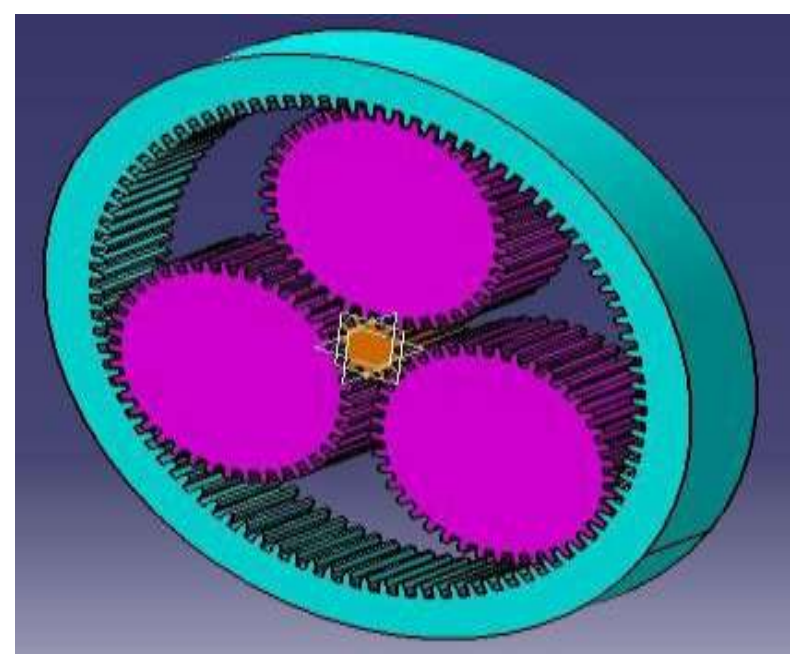

Fig 2 Meshing of Sun,Planet and Annulus gear

Fixed axis spur gears will exhibit lubrication — starvation\| and quickly fail if running at high speeds because the lubricant is slung away. Hence pressurized forced lubrication systems are required for better lubrication. On the other hand, grease lubrication is impractical because of a it's — tunneling effect, in which the grease, over time, is pushed away and cannot flow back into the mesh. In planetary systems the lubricant cannot escape- it is continuously redistributed, - pushed and pulled\| or - mixed\| into the gear contacts, ensuring safe lubrication practically in any mounting position and at any speed. Hence planetary gearboxes can be grease lubricated for the life. This feature is inherent in planetary gearing because of the relative motion between the different gears making up the arrangement. In short the planetary gears systems have high torque density, compact, low inertia and can be grease lubricated for life, which are demands of industrial applications. Hence the author has taken the work on design of compact planetary gear train used in gear boxes used for automation in car parking systems. Next paragraph explains the literature review on the planetary gear design and analysis.

\subsection{WORKING PRINCIPLE}

1. An epicyclic gear train consists of two gears mounted so that the center of one gear revolves around the center of the other. A carrier connects the centers of the two gears and rotates to carry one gear, called the planet gear, around the other, called the sun gear. The planet and sun gears mesh so that their pitch circles roll without slip. A point on the pitch circle of the planet gear traces an epicycloid curve. In this simplified case, the sun gear is fixed and the planetary gear(s) roll around the sun gear.

2. An epicyclic gear train can be assembled so the planet gear rolls on the inside of the pitch circle of a fixed, outer gear ring, which is called as annulus gear. In this case, the curve traced by a point on the pitch circle of the planet is a hypocycloid.

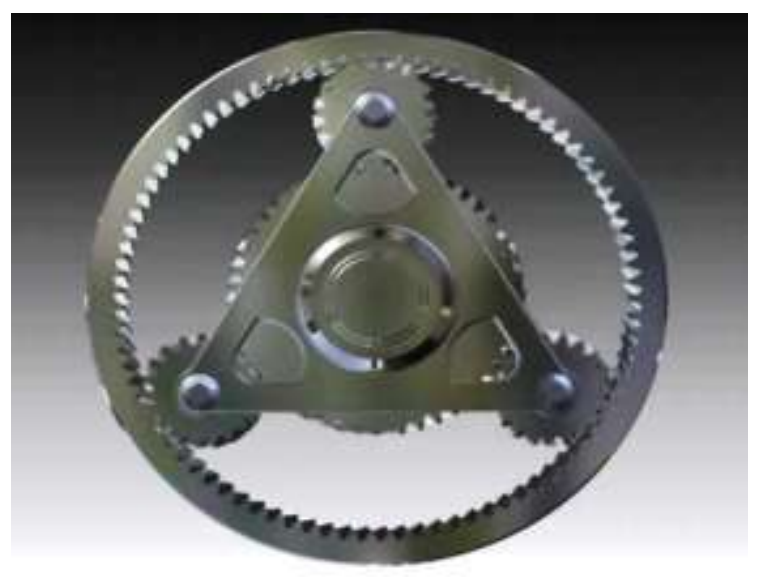

Fig 3 Epicyclic gear 
3. The combination of epicycle gear trains with a planet engaging both a sun gear and an annular gear is called a planetary gear train. In this case, the annular gear is usually fixed and the sun gear is driven.

4. Epicyclic gears get their name from their earliest application, which was the modeling of the movements of the planets in the heavens. Believing the planets, as everything in the heavens, to be perfect, they could only travel in perfect circles, but their motions as viewed from Earth could not be reconciled with circular motion. At around $500 \mathrm{BC}$, the Greeks invented the idea of epicycles, of circles traveling on the circular orbits. With this theory claudius ptolomy in the almagest in $148 \mathrm{AD}$ was able to predict planetary orbital paths. The antykythera mechanism, circa $80 \mathrm{BC}$, had gearing which was able to approximate the moon's elliptical path through the heavens, and even to correct for the nine-year precession of that path.

The gear ratio in an epicyclic gearing system is somewhat non-intuitive, particularly because there are several ways in which an input rotation can be converted into an output rotation. The three basic components of the epicyclic gear are:

* Sun: The central gear

* Planet carrier: Holds one or more peripheral planet gears, of the same size, meshed with the sun gear

* Annulus gear: An outer ring with inward-facing teeth that mesh with the planet gear or gears

In many epicyclic gearing systems, one of these three basic components is held stationary; one of the two remaining components is an input, providing power to the system, while the last component is an output, receiving power from the system. The ratio of input rotation to output rotation is dependent upon the number of teeth in each gear, and upon which component is held stationary.

One situation is when the planetary carrier is held stationary, and the sun gear is used as input. In this case, the planetary gears simply rotate about their own axes at a rate determined by the number of teeth in each gear. If the sun gear has $S$ teeth, and each planet gear has $P$ teeth, then the ratio is equal to $-S / P$. For instance, if the sun gear has 24 teeth, and each planet has 16 teeth, then the ratio is $-24 / 16$, or $-3 / 2$; this means that one clockwise turn of the sun gear produces 1.5 counterclockwise turns of the planet gears.

This rotation of the planet gears can in turn drive the annulus, in a corresponding ratio. If the annulus has $A$ teeth, then the annulus will rotate by $P / A$ turns for each turn of the planet gears. For instance, if the annulus has 64 teeth, and the planets 16 , one clockwise turn of a planet gear results in 16/64, or 1/4 clockwise turns of the annulus. Extending this case from the one above:

- One turn of the sun gear results in $<$ math $>-\mathrm{S} / \mathrm{P}</$ math $>$ turns of the planets

- One turn of a planet gear results in $<$ math $>\mathrm{P} / \mathrm{A}</$ math $>$ turns of the annulus

So, with the planetary carrier locked, one turn of the sun gear results in $<$ math $>$-S/A $</$ math $>$ turns of the annulus.

The annulus may also be held fixed, with input provided to the planetary gear carrier; output rotation is then produced from the sun gear. This configuration will produce an increase in gear ratio, equal to $1+A / S$.

If the annulus is held stationary and the sun gear is used as the input, the planet carrier will be the output. The gear ratio in this case will be $1 /(1+A / S)$. This is the lowest gear ratio attainable with an epicyclic gear train. This type of gearing is sometimes used in tractors and construction equipment to provide high torque to the drive wheels.

In bicycle hub gears, the sun is usually stationary, being keyed to the axle or even machined directly onto it. In this case the gear ratio is simply given by $(\mathrm{S}+\mathrm{A}) / \mathrm{A}$ where $\mathrm{S}$ is the number of teeth on the sun and $\mathrm{A}$ is the number of teeth on the annulus. The number of planet teeth is irrelevant.

Some designs use "compound planets" which have two differently-sized gears on either end of a common casting. The large end engages the sun, while the small end engages the annulus. This may be necessary to achieve smaller step changes in gear ratio when the overall package size is limited. Compound planets have "timing marks" and must be assembled in the correct initial orientation relative to each other, or their teeth will not simultaneously engage the sun and annulus at opposite ends of the planet, leading to very rough running and short life. The use of compound planets is like increasing the size of the annulus; for example, compound planets with teeth in a 2:1 ratio with a 50T annulus would give the same effect as a 100T annulus, but with half the actual diameter.

More planet and sun gear units can be placed in series in the same annulus housing (where the output shaft of the first stage becomes the input shaft of the next stage) providing a larger (or smaller) gear ratio. This is the way some automatic transmission work.

During World War II, a special variation of epicyclic gearing was developed for portable radar gear, where a very high reduction ratio in a small package was needed. This had two outer annular gears, each half the thickness of the other gears. One of these two annular gears was held fixed and had one fewer teeth than did the other. Therefore, several turns of the "sun" gear made the "planet" gears complete a single revolution, which in turn made the rotating annular gear rotate by a single tooth. 


\subsection{DESCRIPTION OF MAJOR COMPONENTS}

\subsubsection{SUN GEAR}

It is the central gear of the epicyclic gearing the output shaft is connected to the sun gear. When it rotates planet gears also rotates and it connects to the input shaft and the output shaft efficiency is more. This gear meshes with and is surrounded by planet gears. The epicyclic gear train family in general has a central "sun" gear which meshes with and is surrounded by planet gears. the sun is usually stationary, being keyed to the axle or even machined directly onto it. In this case the gear ratio is simply given by $(\mathrm{S}+\mathrm{A}) / \mathrm{A}$ where $\mathrm{S}$ is the number of teeth on the sun and $\mathrm{A}$ is the number of teeth on the annulus. The number of planet teeth is irrelevant.

\subsubsection{PLANET GEARS}

Holds one or more peripheral planet gears, all of the same size, meshed with the sun gear. When the sun gear rotates all planet gears rotates at a circular area. In these planet gears carrier is connected from that carrier a shaft taken out . The outermost gear, the ring gear, meshes with each of the planet gears. The more planet gears in the system, the greater load ability and the higher the torque density. The planetary gearing arrangement also creates greater stability (it's a balanced system) and increased rotational stiffness. From these planet gears carrier is connected and the input is given. Power transmission is done through these gears. Because through carrier connected to planets is given as input.

\subsubsection{ANNULUS GEAR}

An outer ring with inward-facing teeth that mesh with the planet gear. It is stationary it will not move .all planet gears are meshes with this annulus gear. If the annulus is held stationary and the sun gear is used as the output, the planet carrier will be the input. The gear ratio in this case will be $1 /(1+\mathrm{A} / \mathrm{S})$. This is the lowest gear ratio attainable with an epicyclic gear train. This type of gearing is sometimes used in tractors and construction equipment to provide high torque to the drive wheels. Three planet gears are meshes and rotated in this annulus gears.

\subsubsection{CARRIER}

The planet gears are held to a cage or carrier that fixes the planets in there orbit relative to each other. In epicyclic gearing system the planet gears are surrounded around the central gear. Those planet gears are connected to the carrier. The input shaft is given to it. In these gearing system three planet gears are connected by the carrier that carrier is connected through three shafts to the planet gears.

\section{DRAWING OF EPICYCLIC GEAR}

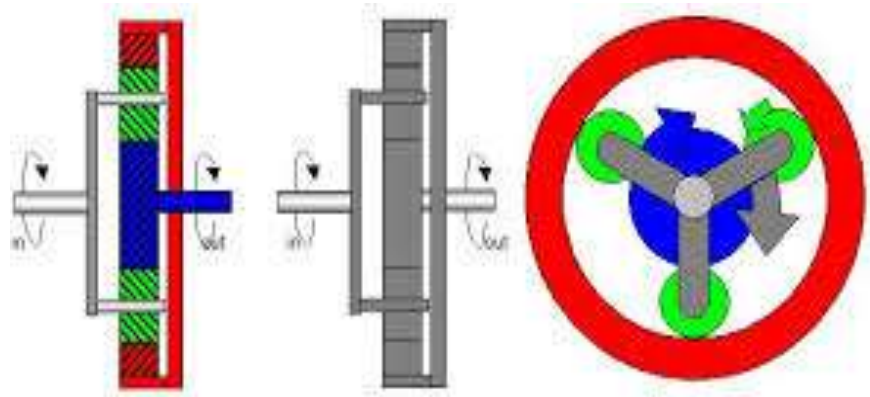

Fig 4 Working of Epicyclic gear

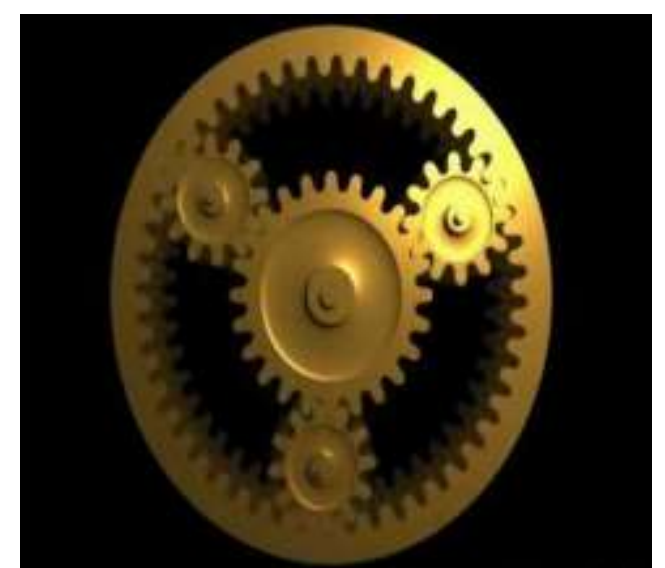

Fig 5 Three dimensional view of epicyclic gear 


\section{DESIGN METHODOLOGY}

The design of planetary gear trains consists of following steps:

A. Determination of reduction ratio for each stage

B. Calculation of number teeth of sun, planets and ring gear.

C. Material and heat treatment Selection.

D. Calculation of contact and bending stress of Gears.

E. Calculation of safety Factors in Hertizen contact stress and bending stress.

\subsection{Determination of Reduction Ratio for each Stage}

The reduction ratio for the given composite arrangement is product of ratios of each stage. Total reduction ratio, $\mathrm{R}=\mathrm{R} 1 * \mathrm{R} 2 * \mathrm{R} 3 * \ldots . . \mathrm{Rn} \ldots$ (1)

Where, R1, R2, R3, ...Rn represents the reduction ratios of each stage of gear train connected in composite.

For easier computation it is preferred that the planetary gear ratio shall be an exact integer $(3,4,6 \ldots)$. As a general practice, ratios used are in decimal system, a ratio of 10:1 is used even though this has no practical advantage for the computer/servo/motion controller. Actually, 10:1 or higher ratios are the weakest; while using the least -balancedll size gears and hence has the lower torque rating. Figure 3 illustrates a cross- section of such a planetary gear arrangement with its central sun gear, multiple planets (3), and the ring gear.

The reduction ratio in planetary gear train is determined as below:-

PCD of Ring Gear + PCD of Sun Gear

Ratio =

\section{PCD of Sun Gear} $\mathrm{z} 1+\mathrm{z} 3$

Ratio $=\frac{----------(3)}{\text { Z3 }}$

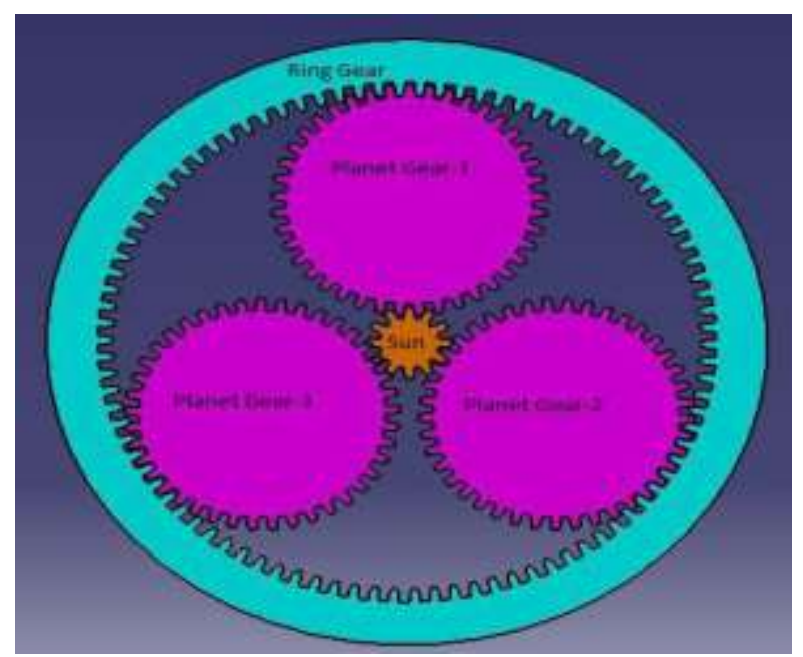

Fig 6 Structure of epicyclic gear

Where, $z 1=$ Number of teeth on Sun Gear.

Z3 = Number of teeth on Ring Gear.

A ratio $2: 1$ is not possible in a simple planetary gear system, since to satisfy the above equation for a ratio of $2: 1$ the sun gear would need to have the same diameter as the ring gear. Figure 7 shows the sun gear size for different ratios. With increased ratio, the sun gear diameter (size) is decreasing. Since gear size effects load ability, the ratio is a strong and direct influence factor for the torque rating.

Figure 7 below shows the gears in a 3:1, 4:1, and 10:1 simple system. At 3:1 ratio, the sun gear is large and the planets are small. The planets are becoming — thin walled\| thus limiting the space for the planet bearings and carrier pins, hence limiting the load ability. The 4:1 ratio is a well balance ratio, with sun and planets having the same size. 5:1 and 6:1 ratios still yield fairly good balanced gear sizes between planets and sun. With higher ratios approaching 10:1, the small sun gear becomes a strong limiting factor for the transferable torque. Simple planetary designs with 10:1 ratios have very small sun gears, which sharply limits torque rating. 

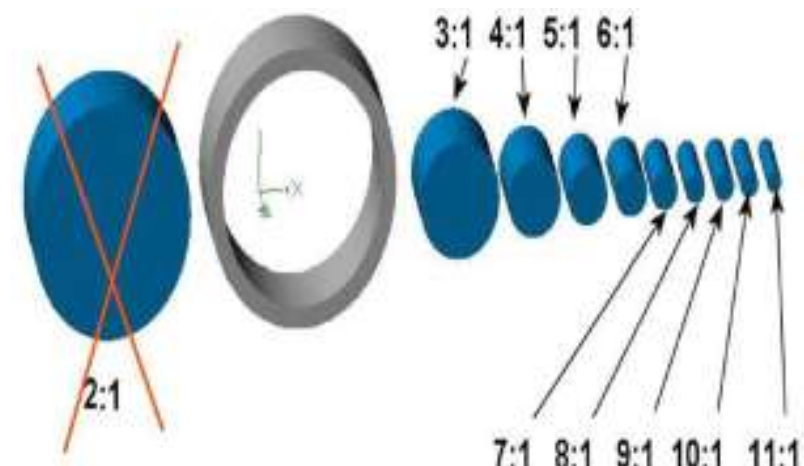

7:1 8:1 9:1 10:1 11:1

Fig- 7: Sun gear size for different ratios

Increasing the number of planets, torque density on gear train will increase. But number planets will be decided by the reduction ratio. If reduction ratio is lower, higher number of planets can be used and in case of higher reduction ratios the planets will interfere with each other. If reduction ratio is greater than 10:1, multiple gears beyond say 3 planets would cause interference.

\section{RESULTS AND DISCUSSION}

The results obtained above are compared on the basis of Size (Volume), weight, and torque density. The detailed are discussed as below:

i) The comparison of volumes of gears in planetary and helical was done and presented in graph-1. Gears were represented by numbers 1,2,3,4,5,6 on $\mathrm{x}$ - axis. In case helical gear system the volume drastically increases as torque increase, whereas this increase was uniform in case of planetary gear system. This increase was very high in last stage gear which transmits the full torque of the transmission system.

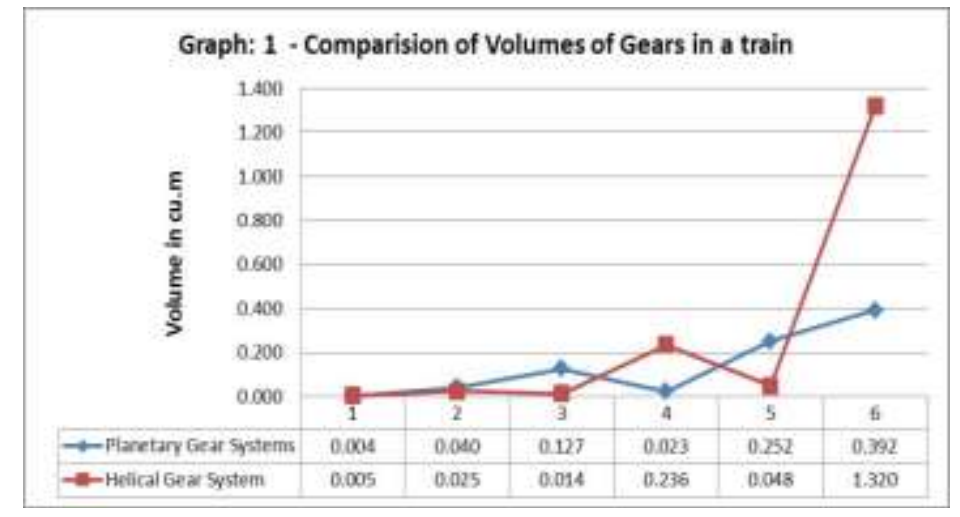

ii) The comparison between weights of gears in planetary and helical gear systems was done and presented in graph2. Gears were represented by numbers $1,2,3,4,5,6$ on $\mathrm{x}$ - axis. In case helical gear system the weight drastically increases for final stage gear which transmits the full torque of the transmission system.

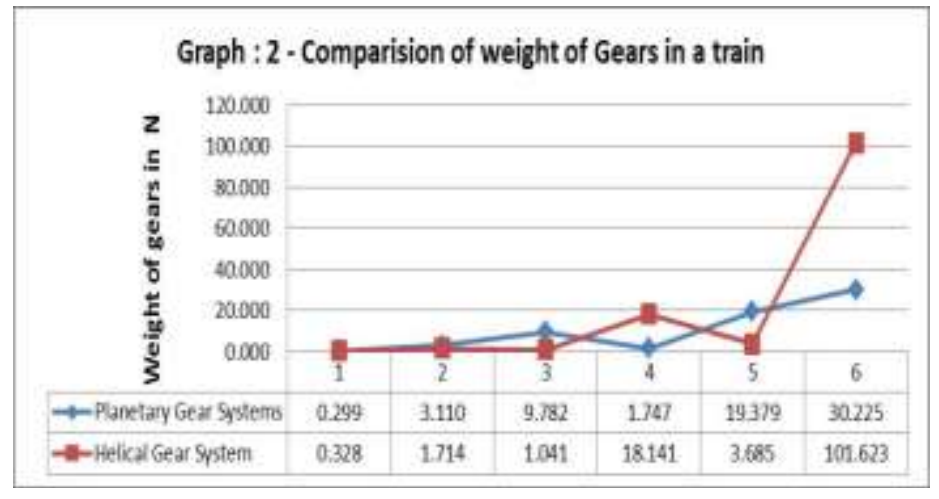




\title{
4.1 APPLICATIONS
}

$\circ$ It is used in military combat vehicles.

$\circ$ It is used in muscle cars.

- It is used in the Antikythera Mechanisms.

- It is used in astronomical clocks.

- It is also used in book stand for proper orientation of books.

- It is used in commercial vehicles and tractors.

\author{
4.2 ADVANTAGES \\ ○ Compactness. \\ ○ High power density. \\ ○ Multiple kinematic combination. \\ $\odot$ Pure torsional reactions. \\ $\odot$ Co axial shafting. \\ - Increased rotational stiffness. \\ ○ Equal distribution of load. \\ $\odot$ Greater stability. \\ ○ Increased torque capability. \\ $\odot$ Outstanding power transmission efficiencies
}

\section{CONCLUSION}

Above study \& results enables us to draw the following conclusion:

1) Reduction ratio $78: 1$ is achieved in 2 stages for planetary gears which was in 3 stage with helical gears, Hence planetary gear system becomes compact as one stages is eliminated.

2) Planetary gears have uniform strength (factor safety) in bending against equivalent helical gears

3) The volume of planetary gear system for the given torque was $49 \%$ less than equivalent helical gear system.

4) The weight of planetary gear system for the given torque was $49 \%$ less than equivalent helical gear system.

5) The torque density (Torque/volume or Torque/ weight) of planetary gear system for the given torque was $96 \%$ higher than equivalent helical gear system.

\section{REFERENCES}

[1]. Lehao Chang, Geng Liu, Liyan WuDr, -A robust modelfor determining the mesh stiffness of cylindrical gearslMechanism and Machine Theory 87 (March-2015) PP: $93-114$

[2]. Dr. Alexander Kapelevich - High Gear Ratio EpicyclicDrives Analysis\|GEAR TECHNOLOGY, June 2014 PP:61-67

[3]. Ovidiu Buiga, Lucian Tudose - Optimal massminimization design of a two-stage coaxial helical speed reducer with Genetic Algorithmsll Advances in Engineering Software 68 (2014) PP: 25-32

[4]. Bernd-Robert Höhn, Karsten Stahl and Philipp Gwinner - Light-Weight Design for Planetary Gear Transmissions\| GEAR TECHNOLOGY, Sept. 2013, PP: 96-103

[5]. Syed Ibrahim Dilawer, Md. Abdul Raheem Junaidi, Dr.S.Nawazish Mehdi —Design, Load Analysis andOptimization of Compound Epicyclic Gear Trains\|American Journal of Engineering Research ISSN 2320-0936 Vol.-02, Issue-10, 2013, PP: 146-153

[6]. Cheon-Jae Bahk, Robert G. Parker - Analyticalinvestigation of tooth profile modification effects on planetary gear dynamicsll Mechanism and Machine Theory.

[7]. Nenad Marjanovic, Biserka Isailovic, Vesna Marjanovic, Zoran Milojevic, Mirko Blagojevic, Milorad Bojic, —A practical approach to the optimization of geartrains with spur gearsl Mechanism and Machine Theory 53 (2012) PP:1-16

[8]. Jelena Stefanović-Marinović and Miloš Milovančević —Load Allocation and Equilibrium for Planetary GearReducers of Earth-PressureBalance Shield MachinellJournal of Mechanics Engineering and Automation 2 (2012) PP: 365-373

[9]. Ulrich Kissling, Inho Bae, -Optimization Procedure forComplete Planetary Gearboxes with Torque, Weight, Costs and Dimensional Restrictionsll Applied Mechanics andMaterials Vol. 86 (2011) pp 51-54.

[10]. Dr.-Ing. T. Schulze, Dipl.-Ing. C. Hartmann, Prof. Dr.- Ing. B. Schlecht, —Calculation of Load Distribution inPlanetary Gears for an Effective Gear Design Process\|American Gear Manufacturers Association October 2010 ISBN: 978--1--55589--983-7 10FTM08 PP: 1-11 